\title{
DuD Recht
}

\section{VerfGH Saarland: Zur Verfassungsmäßigkeit einer Kontaktnachverfolgung}

Die Inanspruchnahme von „Nichtstörern“ ist nach dem Konzept des Infektionsschutzgesetzes rechtlich unbedenklich.

Die Verpflichtung zum Tragen einer Mund-Nasen-Bedeckung in bestimmten, zeitlich und sachlich begrenzten Tagesabschnitten bedarf keiner Ermächtigung durch ein formelles Gesetz und ist angesichts der vorliegenden wissenschaftlichen Stellungnahmen zu ihrer Wirkung ungeachtet des virologischen Streits um ihre Wirksamkeit von der Einschätzungsprärogative der Exekutive gedeckt.

Die Verpflichtung zur Gewährleistung einer Kontaktnachverfolgung durch Erhebung personenbezogener Daten durch Private ist als Eingriff in das Grundrecht auf Datenschutz ohne Vorliegen einer Anlass, Art, Umfang und Verwendung der zu erhebenden persönlichen Informationen bestimmt und normenklar regelnden parlamentarischen gesetzlichen Grundlage verfassungswidrig.

Art. 6 DSGVO enthält vom Vorliegen einer Einwilligung abgesehen keine Befugnis zur Erhebung von personenbezogenen Daten, sondern ausschließlich eine Begrenzung der RechtmäBigkeit der auf anderer Rechtsgrundlage zu erhebenden Daten. Von einer Einwilligung in die Erhebung persönlicher Informationen kann nicht ausgegangen werden, wenn die betroffene Person lediglich die Alternative zwischen ihrer Erteilung und dem Verzicht auf eine Teilnahme am sozialen Leben hat.

(Leitsätze 4 bis 8 des VerfGH Saarland)

Verfassungsgerichtshof des Saarlandes, Beschluss vom 28. August 2020, Az.: Lv 15/20.

Zum Sachverhalt:

Der Beschwerdeführer, ein Rechtsanwalt, wendet sich mit seiner am 05.06.2020 eingegangenen Verfassungsbeschwerde - und dem damit verbundenen Antrag auf Erlass einer einstweiligen Anordnung - gegen einen Beschluss des Oberverwaltungsgerichts des Saarlandes vom 13.05.2020 - 2 B 175/20 -, der seinen Antrag auf Aussetzung des Vollzuges von Bestimmungen früherer Verordnungen der Regierung des Saarlandes zur Bekämpfung der Corona-Pandemie zurückgewiesen hat.

Die von dem Beschwerdeführer im Kern ursprünglich angegriffenen Regelungen der Verordnung vom 02.05.2020 sind nunmehr - in Teilen verändert - in Art. $2 \$ 2$ und $\$ 6$ der Verordnung zur Änderung infektionsrechtlicher Verordnungen zur Bekämpfung der Corona-Pandemie vom 08.08.2020 und - inzwischen vom 21.08.2020 (CP-VO) enthalten.

Das Oberverwaltungsgericht des Saarlandes hat den Antrag auf Außervollzugsetzung von Vorschriften der Verordnung zur Bekämpfung der Corona-Pandemie abgelehnt.
Dagegen wendet sich der Beschwerdeführer mit seiner Verfassungsbeschwerde, mit der er einen Antrag auf Erlass einer einstweiligen Anordnung verbindet.

Aus den Gründen:

A. Die Verfassungsbeschwerde ist nur zum Teil zulässig. [...] 4. Der Beschwerdeführer, der sich nach der Auslegung seines Begehrens gegen die Versagung einstweiligen Rechtsschutzes nämlich die Außervollzugsetzung des Art. $2 \$ 2 \mathrm{Nr}$. 1 bis $4, \S 3$ Abs. 1 Nr. 1 bis 7 und $₫ 6$ der Verordnung in der Fassung vom 02.05.2020 - sowie gegen die Vorschriften der geltenden CP-VO selbst wendet, hat - soweit seine Verfassungsbeschwerde nicht mangels hinreichender Begründung unzulässig ist - plausibel geltend gemacht, in seinen durch die Verfassung des Saarlandes gewährleisteten Grundrechten der allgemeinen Handlungsfreiheit (Art. 2 Satz 1 SVerf), auf Datenschutz (Art. 2 Satz 2 SVerf) und seinem Persönlichkeitsrecht (Art. 2 Satz 1 i.V.m. Art. 1 Satz 1 SVerf) verletzt zu sein.

Eine solche Verletzung ist nicht auszuschließen.

Soweit er anführt, das Oberverwaltungsgericht des Saarlandes habe seinen Justizgewährungsanspruch, das Grundrecht auf effektiven Rechtsschutz und das Willkürverbot verletzt, ist er nicht beschwerdebefugt. Die Frage, ob die von ihm gerügten Grundrechtseingriffe eine parlamentarische gesetzliche Grundlage erforderten, durfte das Oberverwaltungsgericht im Verfahren des einstweiligen Rechtsschutzes angesichts der dafür geltenden Prüfungsmaßstäbe offenlassen. Aus dem gleichen Grund musste es auch den Beweisantritten des Beschwerdeführers in Bezug auf die Wirkung einer Mund-Nasen-Bedeckung nicht nachgehen. $[\ldots]$

B. 1. a. Die Anordnung des $₫ 2$ Abs. 2 CP-VO, bei der Benutzung des öffentlichen Personennahverkehrs, in bestimmten Teilen des öffentlichen Raums und in Einrichtungen der Heilbehandlung eine Mund-Nasen-Bedeckung zu tragen, berührt den Beschwerdeführer in seiner allgemeinen Handlungsfreiheit nach Art. 2 Satz 1 SVerf. Obwohl die Regelung nicht bußgeldbewehrt ist (Art. $2 \S 15 \mathrm{CP}-\mathrm{VO}$ ), enthält sie eine infektionsschutzrechtliche Pflicht, deren Befolgung mit ordnungsrechtlichen Mitteln durchgesetzt werden kann und die dem Beschwerdeführer die freie und unbelastete Teilhabe an zahlreichen Warenangeboten und Dienstleistungen nimmt. Durch das Gebot der Verhüllung eines Teils seines Gesichtes und - wenn auch minimalen - Veränderungen seiner Stimme greift sie auch in den Schutzbereich des durch Art. 2 Satz 1 i.V.m. Art. 1 Satz 1 SVerf gewährleisteten Persönlichkeitsrechts ein.

b. Die grundrechtlichen Beschränkungen des Art. $2 \$ 2 \mathrm{CP}-\mathrm{VO}$ beruhen auf einem - insoweit - verfassungsgemäßen Gesetz.

Rechtsgrundlage von Art. $2 \$ 2$ CP-VO sind die $\$ \$ 28,32$ IfSG. Sie gestatten dem Verordnungsgeber, in Fällen der Feststellung Kranker, Krankheitsverdächtiger, Ansteckungsverdächtiger oder Ausscheider - eines meldepflichtigen Erregers wie des Sars-CoV-2-Virus - die notwendigen Schutzmaßnahmen zu tref- 
fen, soweit und solange dies zur Verhinderung der Verbreitung übertragbarer Krankheiten erforderlich ist.

- Entgegen der Auffassung des Beschwerdeführers hat das Oberverwaltungsgericht des Saarlandes zu Recht angenommen, die von den $\$ \$ 28,32$ IfSG erlaubten Schutzmaßnahmen dürften sich auch gegen „Nichtstörer“, also gegen selbst nicht Kranke, Krankheitsverdächtigte oder Ansteckungsverdächtige und Ausscheider richten.

Schon der Wortlaut des Gesetzes beschränkt die Eingriffsbefugnisse nicht auf einen potenziell gesundheitlich "gefährlichen“ Kreis von Personen. Auch die Systematik gerade des $₫ 28$ Abs. 1 IfSG zeigt [...], dass von den getroffenen Schutzmaßnahmen, etwa bei Veranstaltungen oder in Einrichtungen, notwendigerweise Personen erfasst werden, die selbst nicht krank, krankheits- oder ansteckungsverdächtig sind. Das entspricht auch dem Sinn und Zweck der Normen, die die weitere Ausbreitung übertragbarer Krankheiten auf bislang nicht infizierte Personen verhindern sollen.

- Infektionsschutzrechtliche Maßnahmen müssen daher - sogar typischerweise - infektionsschutzrechtliche „Nichtstörer" erfassen

(so überzeugend Lindner in Schmidt, Covid-19, § 16 Rn. 60 m.w.N.).

Ob die Verordnungsermächtigung des $₫ 32$ IfSG nach Art. 80 Abs. 1 GG zu allen von dem Verordnungsgeber des Saarlandes (und jener anderer Bundesländer) getroffenen Maßnahmen befugt, oder ob nicht Regelungen, die über eine zeitlich begrenzte Einschränkung von Grundrechten besonderen Ranges - wie der Glaubens- und Religionsfreiheit, der Versammlungsfreiheit, der Fortbewegungsfreiheit, der Freizügigkeit oder der Berufsfreiheit - hinaus Grundrechtseingriffe von Gewicht über Monate hinweg erlauben, einer förmlichen - parlamentarischen - gesetzlichen Regelung bedürfen, kann dahinstehen (vgl. insoweit die Frage beispielhaft ansprechend die Entscheidungen des Verfassungsgerichts Brandenburg 03.06.2020 VfGBbg 9/20eA BeckRS 2020, 11248 Rn. 41; VGH Mannheim 05.06.2020 1 S 1623/20 BeckRS 2020, 11786 Rn. 35). Insoweit ist die Verordnung nicht zulässigerweise angegriffen.

Der Vorbehalt des Gesetzes verlangt im Hinblick auf Rechtsstaatsprinzip und Demokratiegebot, dass der Gesetzgeber in grundlegenden normativen Bereichen alle wesentlichen Entscheidungen selbst zu treffen hat und sie nicht dem Handeln und der Entscheidungsmacht der Exekutive überlassen darf. Dabei betrifft die Pflicht zur parlamentarischen Regelung nicht nur die Frage, ob ein bestimmter Gegenstand überhaupt gesetzlich geregelt sein muss, sondern auch, inwieweit diese Regelungen im Einzelnen zu gehen haben. Das hängt vom jeweiligen Sachbereich und der Eigenart des betroffenen Regelungsgegenstands ab (vgl. nur u.a. BVerfG, 27. 11.1990 - 1 BvR 402/87 -, juris, Rn. 39; 24. 09.2003 - 2 BvR 1436/02 -, juris, Rn. 67 f., jeweils m.w.N.). Auch Gesetze, die zu Rechtsverordnungen und Satzungen ermächtigen, können den Voraussetzungen des Gesetzesvorbehalts genügen. Die wesentlichen Entscheidungen müssen aber durch den parlamentarischen Gesetzgeber selbst erfolgen (vgl. OVG Münster zur flächendeckenden Betriebsuntersagung von Verkaufsstellen des Einzelhandels CoVuR 2020, 423).

- Der Beschwerdeführer wendet sich - soweit er die Verpflichtung zum Tragen einer Mund-Nasen-Bedeckung angreift gegen eine vergleichsweise geringe, zeitlich im Tagesablauf vorübergehende und keinen wesentlichen Aufwand erfor- dernde Beschränkung seiner grundrechtlichen allgemeinen Handlungsfreiheit und seines Persönlichkeitsrechts, die das Oberverwaltungsgericht zu Recht als „Unannehmlichkeit“ bezeichnet hat.

Der Grundrechtseingriff betrifft also eine zeitlich auf einen kleinen Bruchteil eines Tages beschränkte Belastung in bestimmten, nicht einmal alltäglich notwendigerweise vorkommenden Situationen. Material, Form und Preis einer „Alltagsmaske“ kann jede betroffene Person selbst bestimmen. Gleiches gilt von der jeweiligen Dauer der Belastung.

- Die grundrechtliche Beschwer ist folglich nicht von einem solchen Gewicht, dass ihre Auferlegung so „wesentlich“ für die Ausübung der allgemeinen Handlungsfreiheit und des Persönlichkeitsrechts wäre, dass sie einer detaillierten formellgesetzlichen Regelung bedürfte.

c. Uneingeschränkt richtig und mit einer die verfassungsrechtlichen Vorgaben sorgfältig und zutreffend berücksichtigenden Begründung hat das Oberverwaltungsgericht des Saarlandes ausgeführt, dass der mit der „Maskenpflicht“ verbundene Eingriff in das Grundrecht der allgemeinen Handlungsfreiheit und in das Persönlichkeitsrecht verhältnismäßig ist, da die Regelung befristet ist, sie sich nicht auf den privaten Bereich erstreckt und für den Träger nur in wenigen, kurzzeitigen Alltagssituationen geringe Belastungen mit sich bringt.

aa. Die Verpflichtung zum Tragen einer Mund-Nasen-Bedeckung verfolgt ein legitimes Ziel, den Schutz Dritter vor der möglichen Viruslast des Pflichtigen beim Ausatmen, Niesen oder Husten, und den Schutz der Gesellschaft insgesamt vor den Folgewirkungen einer Ausweitung der Pandemie.

Soweit der Beschwerdeführer Zweifel zu haben scheint, ob es sich bei Sars-Cov-2 überhaupt um eine ernstliche, den jährlichen Virusinfektionswellen nicht vergleichbare Gefahr für die Gesundheit der Bevölkerung handelt, gilt:

- Zum einen ist es verfassungsrechtlich völlig unerheblich, ob es auch andere Gefahren gibt, die die Gesundheit der Bevölkerung nachhaltig bedrohen, und die zu weniger belastenden staatlichen Maßnahmen führen oder in der Vergangenheit geführt haben.

Schutzpflichten des Staates in Bezug auf die Abwehr einer bestimmten gesundheitlichen Gefahr entfallen nicht, wenn er ihnen irgendwann aus tatsächlichen oder rechtlichen Gründen nicht in Bezug auf andere oder gar alle gesundheitlichen Gefahren entsprechen konnte oder gegenwärtig nicht allen entsprechen kann.

Auch unterliegt es der grundsätzlichen Einschätzungsprärogative der dafür von Verfassungs wegen verantwortlichen staatlichen Organe - dem Parlament und der Regierung - zu entscheiden, welche Bedrohung von einem besonderen, besondere Maßnahmen erfordernden Gewicht ist. Davon abgesehen liegt es indessen bei vernünftiger Betrachtung des globalen Geschehens auf der Hand, dass Sars-Cov-2 aufgrund der bislang weithin fehlenden Instrumente der Prävention, Linderung oder gar Heilung mit anderen viralen Feldzügen nicht vergleichbar ist.

bb. Die Verpflichtung stellt eine grundsätzlich geeignete Maßnahme zur Erreichung dieses Ziels dar.

Zwar wendet der Beschwerdeführer unter Berufung auf Quellen, deren wissenschaftliche Seriosität nicht belegt ist, ein, das Tragen einer Mund-Nasen-Bedeckung sei ungeeignet, Infektionsgefahren zu verringern, es könne sogar der eigenen Gesundheit abträglich sein. 
Soweit einzelnen grundsätzlich validen Stimmen - beispielsweise jener des virologischen Beraters der Regierung des Königreichs Schweden, Tegnell, - Skepsis gegenüber der Anordnung einer Mund-Nasenbedeckung entnommen werden könnte (vgl. verschiedene Meldungen in Medien vom 09./10.08.2020), bedarf es einer genaueren Betrachtung der tatsächlichen Erklärungen: Es ist selbstverständlich, dass das Tragen einer Mund-NasenBedeckung nicht „die Lösung des Problems“ (Tegnell) ist, und dass einem falschen Sicherheitsgefühl allein aufgrund des Tragens einer Maske entgegengewirkt werden muss. Das gilt allerdings auch für die von dem Beschwerdeführer selbst für bedeutsam erklärte Handhygiene. Maßgeblich ist allein, ob eine solche Maßnahme jeweils einen - unter Umständen kleinen - Beitrag dazu leisten kann, infektiöse Verheerungen einzudämmen oder ihnen vorzubeugen.

Soweit sich der Beschwerdeführer darauf beruft, das Material der Mund-Nasen-Bedeckungen könne selbst gesundheitsschädliche Inhalte enthalten, die Maske sei bei unsachgemäßem längeren Gebrauch selbst gesundheitsschädlich und wirke bei Anreicherung mit Schadstoffen kontraproduktiv, trägt seine Argumentation von vornherein nicht.

- Es ist Sache einer jeden Person, sich geeignete Mund-NasenBedeckungen zu beschaffen und sie sachgerecht zu verwenden.

Art. $2 \$ 2$ CP-VO schreibt kein bestimmtes Material der Maske vor; ausführliche, jedermann zugängliche Hinweise erläutern einen richtigen Gebrauch.

Soweit spezifische gesundheitliche Gründe es dem Beschwerdeführer aus objektiver medizinischer - zu attestierender - Sicht dringend angeraten lassen sollten, vom auch nur vorübergehenden Tragen einer Mund-Nasen-Bedeckung abzusehen, ist er von der Pflicht nach Art. $2 \$ 2 \mathrm{CP}-\mathrm{VO}$ ohnehin entbunden.

- Im Übrigen wird in der - seriösen - Wissenschaft ganz überwiegend vertreten, dass das Tragen einer Mund-NasenBedeckung Infektionen mit dem Corona-Virus zwar nicht verhindert und der unmittelbare Selbstschutz nicht belegt ist, dass es jedoch geeignet ist, die Verbreitung der Pandemie einzudämmen, damit die Einrichtungen des Staates und der Gesellschaft, vor allem das Gesundheitssystem, andere und damit mittelbar auch den Träger selbst zu schützen und so einen Beitrag dazu zu leisten, die Notwendigkeit künftiger Grundrechtsbeschränkungen zu verhindern.

Das ergibt sich zunächst aus Stellungnahmen des Robert-KochInstituts als der durch den Gesetzgeber eingerichteten nationalen Behörde zur Vorbeugung übertragbarer Krankheiten sowie zur frühzeitigen Erkennung und Verhinderung der Weiterverbreitung von Infektionen ( $\$ 4$ Abs. 1 IfG). [...]

Dem entspricht die zweite ad-hoc-Stellungnahme der Nationalen Akademie Leopoldina vom 03.04.2020 [...].

Das entspricht auch den internationalen wissenschaftlichen Erkenntnissen und Empfehlungen. [...]

- Angesichts dieser Stellungnahmen ist die durch Art. $2 \$ 2$ Abs. 2 CP-VO getroffene Regelung - deren Eignung ohnehin der gerichtlich nur begrenzt kontrollierbaren Einschätzungsprärogative der Regierung unterfällt - eine geeignete Maßnahme zur Bekämpfung der Ausbreitung der Pandemie und der einander geschuldeten Rücksichtnahme im sozialen Zusammenleben. cc. Die Verpflichtung zum Tragen einer Mund-Nasen-Bedeckung ist auch ein erforderliches Mittel zur Bekämpfung der Ausbreitung der Pandemie. Ein gleich wirksames milderes Mittel ist unter Berücksichtigung der auch insoweit bestehenden Einschätzungsprärogative der Regierung - nicht ersichtlich.

dd. Angesichts der einander gegenüberstehenden Interessen ist die Auferlegung der Pflicht auch angemessen. Die grundrechtliche Beschwer ist gering. Das Tragen einer Mund-Nasen-Bedeckung ist nicht geeignet, den Pflichtigen von der Ausübung grundrechtlicher Freiheiten entscheidend abzuhalten. Die Verpflichtung ist zeitlich eng begrenzt, verlangt einen geringen Aufwand und kann im Wesentlichen als lästig und wenig angenehm betrachtet werden, führt aber nicht zu ins Gewicht fallenden Einschränkungen der Fortbewegungs- und Entfaltungsfreiheit. Auf der anderen Seite leistet sie einen Beitrag zur Abwehr erheblich ins Gewicht fallender Gefahren für Leben, Gesundheit und Freiheit aller sowie der Funktionsweise staatlicher und gesellschaftlicher Einrichtungen.

2. Soweit sich der Beschwerdeführer mit noch hinreichender Begründung gegen die Pflicht zur Gewährleistung einer Kontaktnachverfolgung durch Art. $2 \$ 3 \mathrm{CP}-\mathrm{VO}$ wendet, gilt: Die Norm ist mit Art. 2 Satz 2 der Verfassung des Saarlandes nicht vereinbar. a. Zwar führen die Regelungen zu den Kontaktbeschränkungen, zu denen auch jene der Kontaktnachverfolgung zählt, nicht, wie das Oberverwaltungsgericht völlig zutreffend erkannt hat, zu einem (mittelbaren) Eingriff in die physisch-psychische Integrität im Sinne des gesundheitlichen Wohlergehens und damit nicht zu einem Eingriff in das - von der Verfassung des Saarlandes nicht ausdrücklich verbürgte, sich jedoch aus Art. 1 Satz 1 und Satz 2 SVerf ergebende - Grundrecht auf körperliche Unversehrtheit. Denn die Verfassung schützt die körperliche - physische wie psychische - Integrität. Sie schützt nicht das allgemeine Wohlbefinden im Sinne von Glück, Zufriedenheit und Lebensqualität.

Die Regelung berührt aber den Schutzbereich des Grundrechts auf Datenschutz nach Art. 2 Satz 2 SVerf.

Das Grundrecht gewährleistet die Befugnis des Einzelnen, selbst darüber zu bestimmen, ob, wann, in welchem Ausmaß und wem gegenüber er seine personenbezogenen Daten preisgibt oder deren Verarbeitung gestattet. Denn individuelle Selbstbestimmung als Gewährleistungsgehalt des auch auf der Garantie der Menschenwürde beruhenden Rechts auf freie Entfaltung der Persönlichkeit setzt nicht nur unter den Bedingungen moderner Informationsverarbeitung voraus, dass dem Einzelnen Entscheidungsfreiheit über die Zuordnung von Verhaltensweisen und Geschehnissen zu seiner Person zukommt.

- Wer nicht mit hinreichender Sicherheit überschauen kann, welche ihn betreffende Informationen in bestimmten Bereichen seiner sozialen Umwelt bekannt werden, und wer das Wissen möglicher Kommunikationspartner nicht einigermaßen abzuschätzen vermag, kann in seiner Freiheit wesentlich gehemmt werden, aus eigener Selbstbestimmung zu planen oder zu entscheiden.

Zugleich soll das Grundrecht etwaigen Einschüchterungseffekten entgegenwirken (VerfGH, Urt. v. 21.1.2020 Lv 15/19 m.w.N.; vgl. vor allem BVerfGE 115, 320 (341 f.); BVerfGE 113, 29 (46); grdl. BVerfGE 65, 1 (42 f.).)

Mit der zu Zwecken der Kontaktnachverfolgung geregelten Obliegenheit zur Angabe von Name, Anschrift, (telefonischer oder anderer) Erreichbarkeit und dem Zeitpunkt des Besuchs der be- 
nannten Einrichtungen und Veranstaltungen werden personenbezogene Daten erhoben und verarbeitet.

- b. Die Regelung verpflichtet allerdings den Beschwerdeführer - und andere Grundrechtsträger - selbst nicht, greift also nicht unmittelbar in das Grundrecht auf Datenschutz ein.

- Jedoch kann das die in Art. $2 \$ 3$ Abs. 1 CP-VO genannten Verantwortlichen treffende Gebot, die Kontaktnachverfolgung durch die Erhebung und zeitlich begrenzte Aufbewahrung von personenbezogenen Daten „verpflichtend zu gewährleisten“, Grundrechtsträger mittelbar davon abhalten, diese Einrichtungen und Veranstaltungen - vor allem Gaststätten, Hotels, Gottesdienste, sportliche und kulturelle Ereignisse - zu besuchen.

Von einem grundrechtsrelevanten Eingriff ist auch dann auszugehen, wenn Regelungen das Verhalten einer grundrechtsberechtigten Person mittelbar derart zu beeinflussen vermögen, dass sie von der Ausübung des Grundrechts abgehalten werden kann, wenn sich die „influenzierende Wirkung“ der Regelung (Kloepfer, Verfassungsrecht, Band II, $\$ 51$ Rn. 27) also als funktionales Äquivalent eines imperativen Eingriffs darstellt (BVerfGE 116, 202/222 m.w.N.; vgl. allg. Jarass/Pieroth, GG, 16. Aufl. 2020, Vorb. Vor Art. 1 Rn. 27 ff.). Das trifft auf Art. $2 \$ 3$ CP-VO zu.

Durch diese Regelung werden die Grundrechte der allgemeinen Handlungsfreiheit (Art. 2 Satz 1 SVerf) und, vor allem, auf Schutz der personenbezogenen Daten (Art. 2 Satz 2 SVerf) in einer einem finalen Eingriff gleichkommenden Weise berührt. Der Verordnungsgeber bedient sich nämlich der in Art. $2 \$ 3 \mathrm{CP}$ VO genannten Verantwortlichen als Werkzeugen, um dem - legitimen - Zweck der Bekämpfung der Pandemie durch Feststellung möglicher Infektionsketten und Infektionsverläufe und der Warnung möglicherweise betroffener Personen zu genügen. Er will - verständlicher- und nachvollziehbarerweise - die personenbezogenen Kontaktdaten der Besucherinnen und Besucher von Einrichtungen und Veranstaltungen erfassen, um sie zu warnen und Maßnahmen zu ihrem Schutz und dem Schutz Dritter treffen zu können.

Die Verantwortlichen müssen die Informationen der Personen erheben, aufbewahren und auf Verlangen den Gesundheitsbehörden herausgeben, wollen sie eine bußgeldrechtliche Sanktion (oder gar gewerberechtliche Eingriffe) vermeiden. Das führt dazu, dass Betroffene faktisch lediglich die Möglichkeit haben, auf den Besuch von - beispielsweise - Gaststätten oder Gottesdiensten zu verzichten oder genau diesen Besuch personen- und zeitpunktbezogen zu offenbaren und nicht nur den Verantwortlichen Name, Anschrift und (telefonische oder elektronische) Erreichbarkeit zu benennen, sondern diese Daten auch dem Staat zur Verfügung zu stellen.

Die Erhebung der personenbezogenen Informationen hat ein erhebliches Gewicht.

Ungeachtet der nur durch Interpretation erschließbaren Zweckbindung (die Verordnung nennt die Gründe, die die Gesundheitsbehörden zu einem Herausgabeverlangen berechtigen können, nicht ausdrücklich) werden Grundrechtsberechtigte gehalten, nicht nur in dem Bereich des Besuchs von Gaststätten ihre Kontaktdaten preiszugeben, sondern auch zu dokumentieren, wann sie - beispielsweise - welche Gottesdienste besucht haben und an welchen sonstigen Veranstaltungen und Zusammenkünften sie zu einem bestimmten Zeitpunkt teilgenommen haben.
Das führt nach der gegenwärtigen Praxis im Übrigen nicht nur dazu, dass Verantwortliche und Gesundheitsbehörden über solche Daten verfügen können. Die fehlende normative Ausgestaltung der Kontaktdatenerhebung erlaubt es vielmehr - vor allem im Bereich der Gastronomie -, dass nachfolgende Gäste aufgrund der häufig gebräuchlichen „Ringbucherfassung“ - erkennen und erinnern können, wer vor ihnen das Unternehmen besucht hat und wie er über Telefonnummer, Mailanschrift oder Anschrift erreichbar ist. Art. $2 \$ 3 \mathrm{CP}-\mathrm{VO}$ zwingt also nach seiner gegenwärtigen offenen Ausgestaltung mittelbar dazu, privaten Dritten die eigenen Kontaktdaten und das eigene Verhalten offenzulegen, ohne dass dem auch nur im Geringsten ein infektionsschutzrechtlicher Zweck entnommen werden könnte.

Art. $2 \$ 3$ Abs. 3 CP-VO enthält damit zugleich eine Unterschreitung des Schutzes, den der Staat Betroffenen gegenüber Dritten gewähren muss, wenn er eine Kontaktdatenerhebung bewirkt. Zu dieser Pflicht zum „Datenschutz“ zählen auch normative Vorkehrungen, die infektionsschutzrechtlich möglicherweise gebotene Offenbarung personenbezogener Informationen vor den Augen Dritter geheim zu halten. Auch das trägt zum Gewicht des mittelbaren Eingriffs bei.

Ferner ist - zusätzlich - von Gewicht, dass die pauschale Verweisung in Art. $2 \$ 3 \mathrm{CP}-\mathrm{VO}$ auf „Veranstaltungen“ nach Art. 2 $\$ 6$ CP-VO unklar ist und weder Verantwortlichen noch grundrechtlich Betroffenen deutlich macht, ob auch eine Kontaktnachverfolgung bei Versammlungen (die auch unter den Begriff der von Art. $2 \$ 3 \mathrm{CP}-\mathrm{VO}$ in Bezug genommenen Veranstaltung zählen können) oder bei dem Besuch von gesetzgebenden Körperschaften oder Gerichten gemeint ist. Letztere sind nach Art. $2 \$ 6$ CP-VO nur insoweit vom Anwendungsbereich dieser Vorschrift ausgenommen, als es ihr „Selbstorganisationsrecht“ betrifft. Das bedeutet indessen zunächst nur, dass ihre Beratungen, Verhandlungen und Beschlussfassungen selbst von dem ohnehin der Exekutive nicht durch Verordnung zugänglichen Regelungsbereich erfasst sind. Einer Regierung steht - veranschaulichend gesagt von vornherein nicht das Recht zu, durch Verordnung (auch negativ) zu regeln, wie ein Parlament sich organisiert.

Ob, wovon die Praxis vor allem der Gerichte ausgehen mag, die „Öffentlichkeit“ gleichfalls von der Vorschrift ausgenommen wird - für sie also beispielsweise gar keine quantitativen Begrenzungen und keine Kontaktdatenerhebungen gelten - ist zumindest unklar. Das wiederum könnte dazu führen, dass Parlamente und Gerichte gehalten wären, Kontaktdaten der teilnehmenden Öffentlichkeit zu erfassen. Auf diese Weise könnte - faktisch - die Öffentlichkeit von Verhandlungen gesetz- oder satzungsgebender Körperschaften oder von Gerichten, und damit ein zentrales Element einer transparenten freiheitlichen Demokratie und eines Rechtsstaats, beeinträchtigt werden. Das mag so nicht gemeint und gewollt sein, ist aber nach dem Wortlaut und möglicherweise auch nach dem Zweck der Vorschrift nicht auszuschließen.

Nichts Anderes gilt für die in Art. $2 \$ 6 \mathrm{CP}-\mathrm{VO}$ geregelte Ausnahme für bestimmte politische Veranstaltungen. Zwar regelt Art. $2 \$ 6$ Abs. 6 Satz 2 CP-VO, dass von den Regulierungen des Art. $2 \$ 6$ CP-VO die „Tätigkeit der Parteien und Wählergruppen“ ausgenommen ist. Das ändert allerdings zum einen nichts daran, dass es sich dabei um „Veranstaltungen“ im Sinne des von Art. $2 \$ 3 \mathrm{CP}-\mathrm{VO}$ pauschal in Bezug genommenen Art. $2 \$ 6 \mathrm{CP}-$ VO handelt. Zum anderen sind politische und weltanschauliche, bekenntnisgeprägte Veranstaltungen denkbar, die keine „Tätigkeit" einer politischen Partei oder Wählergruppe darstellen. Die 
Obliegenheit zur Datenangabe kann also auch insoweit zur Folge haben, dass sich Bürgerinnen und Bürger von solchen Veranstaltungen fernhalten. Auch das unterstreicht die Funktionsäquivalenz der Regelung zu einem imperativen Eingriff völlig unabhängig davon, warum für den Bereich des Politischen allein politische Parteien und Wählergruppen von dem Regime des Art. 2 $\$ 3$ und $\$ 6 \mathrm{CP}-\mathrm{VO}$ gesondert ausgenommen sind.

In jedem Fall birgt die Regelung im Übrigen - auch wenn das so gewiss auch nicht beabsichtigt sein mag - die Gefahr, dass Bewegungs- und Persönlichkeitsprofile von Grundrechtsträgern erstellt werden können, ohne dass zugleich verfahrensrechtliche Regelungen zur Missbrauchsabwehr getroffen worden wären. Die Kontaktdatenerhebung und -nachverfolgung erlaubt nämlich grundsätzlich, so schwierig das bei einer gegenwärtig nur händischen Erhebung sein mag, zu recherchieren, wann sich Betroffene wo aufgehalten haben, ob und in welcher Häufigkeit sie Gaststätten besucht haben, ob und wenn ja welche Gottesdienste sie besucht haben und bei welchen gesellschaftlichen oder politischen Zusammenkünften sie anwesend waren.

Es steht daher außer Frage, dass Art. $2 \$ 3 \mathrm{CP}-\mathrm{VO}$ damit in die Grundrechte von Bürgerinnen und Bürgern eingreift.

c. Der Eingriff ist - auf der Grundlage der derzeitigen Verordnungsregelung - nicht gerechtfertigt. Ihm fehlt eine tragfähige gesetzliche Grundlage.

aa. Ein Eingriff in das Grundrecht auf Schutz der personenbezogenen Daten bedarf einer in der Regel förmlichen, parlamentarischen (vgl. zuletzt VerfGH 21.01.2020 Lv 15/19 unter B 3 c) Ermächtigung, die die zu erhebenden personenbezogenen Daten als solche, den Anlass und den spezifischen Zweck der Erhebung, die Art und Dauer der Aufbewahrung sowie ihre Löschung normenklar und bestimmt regelt und den Grundsatz der Verhältnismäßigkeit wahrt (vgl. zuletzt BVerfG 27.05.2020 1 BvR 1873/13 u.a.; BVerfGE 65, 1 ff. (44 ff., 151 ff.)). Schon daran fehlt es.

bb. Das IfSG enthält keine solche Ermächtigung. Alleine die sich aus $₫ 28$, $\$ 32$ IfSG ergebende Befugnis, „die notwendigen Schutzmaßnahmen zu treffen“, ist - jedenfalls für den generell-abstrakten Eingriff in das Recht auf informationelle Selbstbestimmung - zu unbestimmt. Art. $2 \$ 3 \mathrm{CP}-\mathrm{VO}$ ist - abgesehen davon, dass die Norm lediglich rudimentäre Regelungen über den Zweck der Datenerhebung und den Umgang mit Daten enthält - lediglich exekutives Recht.

cc. Von den formellen Voraussetzungen eines Eingriffs in das Grundrecht auf Datenschutz ist nicht deshalb eine Ausnahme geboten, weil sie aus Anlass der Gefahren der automatisierten Datenverarbeitung entwickelt worden sind. Die Entwicklung der Digitalisierung zeigt zwar ein besonderes Gefährdungspotenzial von Eingriffen in das Grundrecht auf Datenschutz. Sie befreit den parlamentarischen Gesetzgeber aber nicht in Fällen einer Datenerhebung in der „analogen“ Welt, selbst hinreichend bestimmte Regelungen zur Beschaffung und Nutzung personenbezogener Informationen zu treffen. Art. 2 Satz 2 SVerf unterscheidet nicht zwischen automatisiert verarbeiteten und nicht-automatisiert verarbeiteten Daten. Im Übrigen ist nicht von der Hand zu weisen, dass zunächst händisch dokumentierte Informationen jederzeit - wie die innovative Entwicklung einer App für Gästelisten durch ein saarländisches Unternehmen zeigt (Saarbrücker Zeitung vom 21.08.2020 A 7) - digitalisiert und damit auch den Gefahren der automatisierten Datenverarbeitung zugänglich werden können, ohne dass die betroffene Person davon erfährt oder dem entgegenwirken kann. dd. Das Erfordernis einer parlamentarischen gesetzlichen Grundlage ist auch keine verzichtbare bloße Formalität. Während Verordnungen wie jene zur Bekämpfung der Corona-Pandemie bis zur ihrer Veröffentlichung im Wesentlichen im Internum der Exekutive erarbeitet, beraten und beschlossen werden, und Bürgerinnen und Bürger damit vor die vollendete und geltende Regelung gestellt werden, gewährleistet ein parlamentarisches Gesetz die Debatte von Für und Wider vor dem Forum der Öffentlichkeit und damit ein wesentliches Element der repräsentativen Demokratie. Daher mag in einer Notsituation, in der kurzfristiges Handeln einer Regierung zwingend erscheint, die Verordnung auf der Grundlage einer hinreichend bestimmten Ermächtigung ein notwendiges und wichtiges Instrument der Staatsleitung sein. Je länger grundrechtliche Belastungen von Bürgerinnen und Bürgern indessen andauern, desto wichtiger wird es indessen, die Regelung ihrer Grundlagen und Grenzen dem ohnehin originär verantwortlichen parlamentarischen Gesetzgeber zu überlassen.

ee. Die Datenerhebung findet ihre Rechtfertigung nicht in einer „Einwilligung“ Betroffener.

- Selbst wenn man jeweils davon ausgehen würde, dass Betroffene mit der Angabe ihrer Kontaktdaten im Interesse der Sache formal einverstanden sein sollten, rechtfertigt eine solche Einwilligung die Datenerhebung nicht, weil sie nur um des Preises der Versagung einer Teilhabe am gesellschaftlichen, politischen und religiösen Leben verweigert werden könnte. Daher hat die Verfassungsrechtsprechung die verfassungsrechtlich nicht unähnliche Frage, ob dem generellen Verbot des Rauchens in Gaststätten entgegenstehe, dass Gäste „Rauchergaststätten“ freiwillig aufsuchten, klar mit der - auch im Streitfall tragenden - Begründung verneint, eine solche Auffassung würde dazu führen, Bürger von der Teilhabe an sozialer Interaktion faktisch auszuschließen.

ff. Entgegen der Auffassung der Beteiligten stellt Art. 6 DSGVO keine ausreichende Ermächtigung dar.

Das folgt zunächst schlicht bereits daraus, dass die Verordnung Art. 6 DSGVO gar nicht als Rechtsgrundlage ihres Erlasses nennt. Nach Art. 104 SVerf, der Art. 80 Abs. 1 Satz 2 GG entspricht, ist jedoch - als Wirksamkeitsvoraussetzung einer Rechtsverordnung - ihre Rechtsgrundlage in der Verordnung selbst anzugeben. Schon daran fehlt es.

Im Übrigen würde es ein grundlegendes Missverständnis des Art. 6 DSGVO darstellen, sähe man darin eine beliebige, rechtsformunabhängige Befugnis zur Erhebung von Daten unter den dort genannten Voraussetzungen. Art. 6 DSGVO begrenzt die Befugnis zur Datenerhebung, sie gewährt sie nicht.

Art. 6 DSGVO regelt die Rechtmäßigkeit der Verarbeitung von Daten. Dabei bestimmt ihr Abs. 1 Satz 1 sieben verschiedene Bedingungen, unter denen sie erlaubt ist. Neben dem Vorliegen einer Einwilligung betroffener Personen (lit. a ) sind es - im Streitfall bedeutsam - die Erfüllung einer rechtlichen Verpflichtung, der der Verantwortliche unterliegt (lit. c), ihre Erforderlichkeit zum Schutz lebenswichtiger Interessen Betroffener oder Dritter (lit. d) oder ihre Erforderlichkeit zur Wahrnehmung einer Aufgabe im öffentlichen Interesse (lit. e). Diese, beispielhaft genannten, Rechtmäßigkeitsvoraussetzungen stellen aber nicht zugleich Eingriffsgrundlagen dar, sondern bedürfen einer speziellen unionsrechtlichen oder mitgliedschaftlichen Norm, die die Datenerhebung als solche - in den Grenzen des Art. 6 DSGVO - 
vorsieht oder gestattet (vgl. nur Kühling/Bucher, DSGVO, Art. 6 Rn. 78; BeckOK-DSGVO/Wolff/Brinck Art. 6 DSGVO Rn. 14).

Das lässt sich zunächst aus Art. 6 Abs. 3 DSGVO selbst erschließen, der gerade zu den Rechtmäßigkeitsvoraussetzungen der Buchstaben c und e des Absatzes 1 auf die Quellen verweist, die die „Rechtsgrundlage“ für die Verarbeitung festlegen können und zu ihnen Näheres bestimmt. Wäre Rechtsgrundlage Art. 6 Abs. 1 Satz 1 lit. c und e selbst, bedürfte es dieser Vorschrift nicht, oder sie müsste als zusätzliche Rechtmäßigkeitsvoraussetzung formuliert sein.

Es folgt - beispielsweise - weiter aus Erwägungsgrund 45 der DSGVO. Danach setzt eine Verarbeitung von Daten zur Wahrnehmung einer Aufgabe im öffentlichen Interesse eben nicht nur voraus, dass diese Voraussetzungen materiell vorliegen, sondern dass eine gesetzliche Grundlage des Unionsrechts oder des mitgliedstaatlichen Rechts für eine Verarbeitung - die dann den Schranken des Art. 6 DSGVO genügen muss - vorliegt. Auch wenn Erwägungsgrund 41 der DSGVO darauf hinweist, dass die Mitgliedstaaten frei sind, die konkrete Form von Rechtsgrundlagen einer Erhebung personenbezogener Daten zu regeln, zeigt das zunächst, dass es solcher Rechtsgrundlagen als Befugnisnormen für eine Erhebung von Daten selbst bedarf. Zugleich macht Erwägungsgrund 41 der DSGVO deutlich, dass die Form der Ermächtigung - förmliches Gesetz oder Verordnung - von dem Verfassungsrecht der Mitgliedstaaten bestimmt wird (vgl. u.a. Ehmann/ Selmayr, DSGVO Art. 6 Rn. 40). Das gilt auch, soweit Art. 6 Abs. 1 Satz 1 lit. d DSGVO und Erwägungsgrund 46 der DSGVO die Verarbeitung von personenbezogenen Daten in Fällen epidemischer Entwicklungen für rechtmäßig erklären.

Dem allen kann auch nicht entgegengehalten werden, dass Art. $2 \$ 3$ Abs. 3 2. Hs. CP-VO die Löschung der Daten „gemäß der geltenden Datenschutzgrundverordnung“ anordnet. Zum einen bezieht sich die Regelung nämlich ausschließlich auf die Löschung, nicht aber auf die Erhebung und Nutzung der Daten. Zum anderen „gilt“ die Datenschutzgrundverordnung nur für die ganz oder teilweise automatisierte Verarbeitung personenbezogener Daten sowie für die nichtautomatisierte Verarbeitung personenbezogener Daten, die in einem Dateisystem gespeichert sind oder gespeichert werden sollen. Die Kontaktdaten bei dem Besuch der in Art. $2 \$ 6 \mathrm{CP}-\mathrm{VO}$ genannten Einrichtungen und Veranstaltungen werden - soweit ersichtlich und mangels anderer gesetzlicher Anordnung - gegenwärtig handschriftlich angegeben und in dieser Form gesammelt und aufbewahrt. Daher liegt keine automatisierte Verarbeitung von Daten und auch keine Verarbeitung von Daten, deren Speicherung in einem Dateisystem beabsichtigt ist (Art. 2 Abs. 1 DSGVO), vor. Die Regelung ist daher zusätzlich geeignet, die Intensität des Eingriffs zu verharmlosen und zu verunklaren.

gg. Ob das formelle Erfordernis einer parlamentsgesetzlichen Regelung der Datenverarbeitung auch dann gilt, wenn betroffene Personen für einen vorübergehenden Zeitraum in einer Situation katastrophischen Ausmaßes zur Offenbarung ihrer personenbezogenen Daten nicht selbst verpflichtet und notfalls zu ihr gezwungen werden können, sondern ihnen zeitlich begrenzt die Wahl zwischen einer zur Preisgabe ihrer Daten führenden Teilnahme an bestimmten Angeboten oder einem Verzicht auf diese Inanspruchnahme eröffnet wird, kann dahinstehen. Denn ungeachtet der jeweils überschaubaren zeitlichen Begrenzung der Gewährleistung einer Kontaktnachverfolgung durch die Regelungen zur Bekämpfung der Pandemie kann nicht verkannt werden, dass der durch Art. $2 \$ 3 \mathrm{CP}-\mathrm{VO}$ ermöglichte Eingriff bereits längere Zeit andauert und angesichts der Infektionslage voraussichtlich weitere Monate auf der Grundlage einer bloßen Rechtsverordnung andauern würde.

- Damit ist der mittelbare Eingriff in das Grundrecht auf Datenschutz inzwischen jedenfalls von einer derartigen Intensität, dass nur ein parlamentarisches Gesetz ihn und seine Voraussetzungen und Grenzen rechtfertigen könnte.

hh. Soweit schließlich eingewandt werden könnte, das Instrument der Verordnung erlaube in Zeiten katastrophischer Geschehnisse eine höhere Flexibilität der Gefahrenabwehr, die angesichts des sich möglicherweise rasant verändernden Infektionsgeschehens notwendig sei, könnte der Verfassungsgerichtshof diese Auffassung nicht teilen. Denn auch auf der Grundlage einer ausreichend normklaren und hinreichend bestimmten gesetzlichen Ermächtigung zur Verarbeitung personenbezogener Daten könnte - bei entsprechender Nutzung der verfassungsrechtlichen Instrumente - die nötige Flexibilität exekutiver Reaktionen unschwer erhalten bleiben, also die jederzeitige kurzfristige Reaktion auf neue stärkere oder geringere Infektionsverläufe erlaubt werden.

d. Da Art. 2 \& $3 \mathrm{CP}-\mathrm{VO}$ allerdings einem uneingeschränkt legitimen Ziel dient und die bisherige - bundesweite - Rechtsprechung zwar immer wieder das Problem einer ausreichenden gesetzlichen Ermächtigung angesprochen, dies jedoch im Rahmen von Entscheidungen über Anträge auf einstweilige Außervollzugsetzung von verordnungsrechtlichen Normen dahingestellt gelassen hat, macht der Verfassungsgerichtshof von der Möglichkeit Gebrauch, die mit der Verfassung unvereinbare Vorschrift vorübergehend unter Normierung verfahrensrechtlicher Sicherungen des Grundrechts in Kraft zu lassen und dem Landtag des Saarlandes eine angemessene Frist zur Abhilfe zu setzen.

- Die bloße Unvereinbarkeitserklärung, verbunden mit der Anordnung befristeter Fortgeltung der verfassungswidrigen Regelung, ist zulässig, weil die sofortige Ungültigkeit der beanstandeten Norm des Art. 2 \$ CP-VO dem Schutz überragender Güter des Gemeinwohls teilweise die Grundlage entziehen würde und eine Abwägung mit den betroffenen Grundrechten ergibt, dass der Eingriff für eine Übergangszeit hinzunehmen ist; der Gesetzgeber erhält so die Gelegenheit, eine gesetzliche Regelung zu treffen

(vgl. BVerfGE 109, 190 [235 f.]; auch bereits BVerfGE 33, 1 [13]; 33, 303 [347 f.]; 40, 276 [283]; 41, 251 [266 ff.]; 51, 268 [290 ff.]). Die in Art. $2 \$ 3$ CP-VO ausgesprochene Verpflichtung leistet einen Beitrag zur Bekämpfung der Ausbreitung der Pandemie und damit zur Abwehr erheblich ins Gewicht fallender Gefahren für Leben, Gesundheit und Freiheit aller sowie der Funktionsweise staatlicher und gesellschaftlicher Einrichtungen. Sie dient damit einem überragenden Gemeinschaftsinteresse. Diesen Schutz zu gewährleisten ist Aufgabe des Staates. Trotz des Fehlens einer hinreichenden parlamentarischen Ermächtigung ergibt die Abwägung mit dem Grundrecht auf Datenschutz, dass der mittelbare Eingriff in dieses Grundrecht von den Betroffenen für eine Übergangszeit hinzunehmen ist. Auch das Bundesverfassungsgericht hatte in seiner Rechtsprechung eine solche Unvereinbarkeitserklärung bereits entwickelt, als sie im BVerfGG ( $\$ 31$ Abs. 2 Sätze 2 und 3) noch nicht vorgesehen war. [...] 


\section{BGH: Zugang der Erben zum vollständigen Facebook-Konto}

Zur Auslegung eines Vollstreckungstitels (siehe BGH, Urteil vom 12. Juli 2018 III ZR 183/17, BGHZ 219, 243), der die ein soziales Internet-Netzwerk betreibende Schuldnerin verpflichtet, den Erben einer verstorbenen Teilnehmerin an dem Netzwerk Zugang zu dem vollständigen Benutzerkonto und den darin vorgehaltenen Kommunikationsinhalten der Erblasserin zu gewähren.

Bundesgerichtshof, Beschluss vom 27. August 2020, Az.: III ZB 30/20.

\section{Zum Sachverhalt:}

Die Parteien streiten über den Zugang zum Benutzerkonto eines sogenannten sozialen Netzwerks, das die Beklagte betreibt. Die Gläubigerin hat beansprucht, Zugang zu dem bei der Schuldnerin unterhaltenen Konto ihrer verstorbenen minderjährigen Tochter und den darin vorgehaltenen Kommunikationsinhalten zu erhalten. Sie ist neben deren Vater Mitglied der Erbengemeinschaft.

Das Landgericht hat die Schuldnerin am 17. Dezember 2015 verurteilt, der Erbengemeinschaft Zugang zu dem vollständigen Benutzerkonto der Erblasserin und den darin vorgehaltenen Kommunikationsinhalten zu gewähren. Auf die Berufung der Schuldnerin hat das Kammergericht das Urteil des Landgerichts abgeändert und die Klage abgewiesen. Auf die Revision der Gläubigerin hat der Senat mit Urteil vom 12. Juli 2018 das Urteil des Kammergerichts aufgehoben und die Berufung der Beklagten gegen das Urteil des Landgerichts zurückgewiesen.

Die Schuldnerin hat am 30. August 2018 der Gläubigerin einen USB-Stick übermittelt, der eine PDF-Datei mit mehr als 14.000 Seiten enthält, die nach den Angaben der Schuldnerin eine Kopie der ausgelesenen Daten aus dem von der Verstorbenen bei der Schuldnerin geführten Konto enthält. Zwischen den Parteien ist streitig, ob hierdurch die Verpflichtung der Schuldnerin aus dem Urteil des Landgerichts vom 17. Dezember 2015 erfüllt ist und inwieweit die auf dem USB-Stick enthaltenen Daten strukturiert angeordnet sind.

Auf Antrag der Gläubigerin hat das Landgericht wegen Nichterfüllung der Verpflichtung aus seinem Urteil vom 17. Dezember 2015 gegen die Schuldnerin ein Zwangsgeld von $10.000 €$ festgesetzt. Auf die sofortige Beschwerde der Schuldnerin hat das Kammergericht den Beschluss des Landgerichts aufgehoben und den Antrag der Gläubigerin auf Festsetzung eines Zwangsmittels gegen die Schuldnerin zurückgewiesen.

Mit ihrer vom Kammergericht zugelassenen Rechtsbeschwerde begehrt die Gläubigerin die Wiederherstellung der landgerichtlichen Entscheidung.

\section{Aus den Gründen:}

Die nach $₫ 574$ Abs. 1 Satz 1 Nr. 2 ZPO statthafte und auch im Übrigen zulässige Rechtsbeschwerde der Gläubigerin ist begründet. 1. Das Beschwerdegericht hat ausgeführt, die Schuldnerin sei mit der Übermittlung des USB-Sticks ihrer Verpflichtung, den Zugang zu dem vollständigen Benutzerkonto der Erblasserin und den darin vorgehaltenen Kommunikationsinhalten zu gewähren, hinreichend nachgekommen, so dass die Verhängung eines Zwangsmittels nach $\$ 888 \mathrm{ZPO}$ nicht gerechtfertigt sei. [...]
2. Das hält der rechtlichen Nachprüfung nicht stand. Die Voraussetzungen zur Festsetzung eines Zwangsmittels gemäß $\$ 888$ Abs. 1 Satz 1 ZPO liegen vor.

- Entgegen der Auffassung des Beschwerdegerichts hat die Schuldnerin mit der am 30. August 2018 erfolgten Übermittlung eines USB-Sticks an die Gläubigerin ihre Verpflichtung aus dem rechtskräftigen Urteil des Landgerichts vom 17. Dezember 2015, der Erbengemeinschaft Zugang zu dem vollständigen Benutzerkonto und den darin vorgehaltenen Kommunikationsinhalten zu gewähren, nicht erfüllt.

a) Der Zugang zu dem vollständigen Benutzerkonto beinhaltet die Möglichkeit der Gläubigerin, vom Konto und dessen Inhalt auf dieselbe Art und Weise Kenntnis nehmen zu können, wie dies die Erblasserin konnte.

- Das bedeutet, dass sich die Gläubigerin in dem Benutzerkonto mit Ausnahme einer aktiven Nutzung so „bewegen“ können muss wie zuvor die Erblasserin selbst.

Eine entsprechende Verpflichtung ergibt sich bereits aus dem Tenor des Vollstreckungstitels (nachfolgend zu bb), jedenfalls aber aus dessen Entscheidungsgründen und den Entscheidungsgründen des Senatsurteils vom 12. Juli 2018 (nachfolgend zu cc).

aa) Das Vollstreckungsgericht hat durch Auslegung des Vollstreckungstitels zu ermitteln, welche Verhaltensweisen dieser erfasst. [...]

bb) Vorliegend ergibt bereits die Auslegung des Tenors des Vollstreckungstitels, wie das Landgericht zutreffend erkannt hat, dass der Gläubigerin durch die Schuldnerin nicht nur Zugang zu den im Benutzerkonto vorgehaltenen Kommunikationsinhalten zu gewähren, sondern darüber hinaus auch die Möglichkeit einzuräumen ist, vom Benutzerkonto selbst und dessen Inhalt auf dieselbe Art und Weise Kenntnis nehmen zu können, wie dies die Erblasserin konnte.

- Die im Tenor ausgesprochene Verpflichtung der Schuldnerin, der Gläubigerin „Zugang“ zu dem vollständigen Benutzerkonto zu gewähren, weist schon sprachlich darauf hin, dass die Gläubigerin in das im Herrschaftsbereich der Schuldnerin befindliche Konto „hineingehen“ können muss und ihr nicht lediglich dessen Inhalte zu übermitteln sind (zutreffend Braun, jurisPR-ITR 15/2019 Anm. 3 Buchst. C; Pruns aaO S. 247).

Dieses Verständnis wird durch die kumulative Nennung der Gewährung sowohl des Zugangs zum vollständigen Benutzerkonto als auch zu den dort vorgehaltenen Kommunikationsinhalten im Tenor bestätigt. Daraus folgt, dass der Zugang allein zu den Kommunikationsinhalten nicht genügt, sondern mehr, nämlich der Zugang zum Konto selbst, zu gewähren ist (so auch Pruns aaO). cc) Aus den Entscheidungsgründen des Urteils des Landgerichts vom 17. Dezember 2015 und des Senatsurteils vom 12. Juli 2018 ergibt sich ebenfalls, dass der Zugang zu dem vollständigen Benutzerkonto, zu dessen Gewährung die Schuldnerin verurteilt worden ist, auch die Möglichkeit der Gläubigerin beinhaltet, vom Benutzerkonto und dessen Inhalt auf dieselbe Art und Weise Kenntnis nehmen zu können, wie dies die Erblasserin konnte. Die Gläubigerin muss sich in dem Benutzerkonto mit Ausnahme einer aktiven Nutzung so „bewegen“ können wie zuvor die Erblasserin selbst.

(1) (a) Nach den Ausführungen des Landgerichts in dessen Urteil vom 17. Dezember 2015 ist das Recht der Erblasserin, Zugang zu dem Nutzerkonto zu haben und auf die Server der Beklagten zuzugreifen, zusammen mit dem mit der Beklagten bestehenden 
Vertragsverhältnis im Wege der Gesamtrechtsnachfolge gemäß $\$ 1922$ BGB auf ihre Erben übergegangen. Der Erbengemeinschaft stehe aus dem auf sie gemäß $\$ 1922$ BGB übergegangenen Vertrag ein Anspruch dergestalt zu, dass die Beklagte ihr Zugang zu dem Benutzer-Account der Erblasserin zu verschaffen habe (LG Berlin, ZUM-RD 2016, 471, 473 f., 478).

- Aus dieser erbrechtlichen Herleitung des Anspruchs der Erben folgt unmittelbar, dass sie nicht schlechter gestellt werden dürfen als die Erblasserin, deren Rechte auf sie gemäß $\$ 1922$ BGB im Wege der Universalsukzession übergegangen sind, und dass ihnen daher als (neuen) Vertragspartnern der Beklagten ein nach Art und Weise identischer Zugang zu dem Benutzerkonto zu gewähren ist wie der Erblasserin selbst (so auch Biermann, ErbR 2020, 343, 344).

Im weiteren Verlauf seiner Ausführungen vertritt das Landgericht die Auffassung, es stelle eine unangemessene Benachteiligung der Erben gemäß $\$ 307$ Abs. 1, Abs. 2 Nr. 1 BGB dar, wenn nach den Nutzungsbedingungen der Beklagten eine beliebige Person der Freundesliste eine Versetzung des Profils in den Gedenkzustand veranlassen könne und dann eine Anmeldung des Kontos selbst mit gültigen Zugangsdaten für die Erben nicht mehr möglich sei (aaO S. 475). Auch hieraus ist erkennbar, dass das Landgericht auf einen Anspruch der Klägerin auf Zugang zu dem Benutzerkonto mittels der gültigen Zugangsdaten und damit auf einen Zugang erkannt hat, wie er gemäß dem Nutzungsvertrag mit der Beklagten auch der Erblasserin zur Verfügung stand.

Die weitere Feststellung des Landgerichts, der von ihm bejahte Auskunftsanspruch der Erben nach $₫ 34$ BDSG gehe auf Zugangsgewährung, denn die Beklagte müsse nicht selbst referieren, was in dem Account der Erblasserin stehe (aaO S. 477), lässt ebenfalls erkennen, dass das Gericht zwischen der Übermittlung des Inhalts des Benutzerkontos (Referieren durch die Beklagte) und dem von der Beklagten zu gewährenden Zugang zu dem Konto bewusst unterschieden hat.

(b) Soweit die Rechtsbeschwerdeerwiderung ausführt, aus der zur Auslegung des Vollstreckungstitels heranzuziehenden prozessualen Vorgeschichte ergebe sich, dass die Gläubigerin lediglich die Inhalte des Benutzerkontos habe zur Kenntnis nehmen wollen, aber nicht an dessen darüber hinausgehender Funktionalität interessiert gewesen sei, erschließt sich dies aus den von ihr in Bezug genommenen Schriftsätzen der Gläubigerin nicht.

Das gilt vor allem für die Klageschrift vom 24. April 2015. Aus ihr wird vielmehr eindeutig erkennbar, dass die Klägerin (und jetzige Gläubigerin) nicht nur die Kenntnis von den Inhalten des Benutzerkontos, sondern auch den vollen Zugriff auf das Konto selbst begehrt hat. [...]

Soweit die Klägerin in ihrer Berufungserwiderung vom 10. Februar 2017 (S. 5) ausgeführt hat, die Beklagte verfüge über mindestens zwei Funktionen, die eine Zugangsgewährung ermöglichten, die dem Tenor des Urteils des Landgerichts gerecht werde, zum einen die Entsperrung des Nutzerprofils und zum anderen den Download des vollständigen Benutzerkontos als DateiContainer, kann hieraus nicht geschlossen werden, dass die Klägerin nunmehr in Abkehr von ihrem erstinstanzlichen Begehren allein durch die Übermittlung eines Datei-Containers die ausgeurteilte Verpflichtung der Beklagten als erfüllt ansah. Selbst wenn indes ihre Ausführungen so zu verstehen sein sollten, könnte nicht davon ausgegangen werden, dass die Klägerin mit dem „Download des vollständigen Benutzerkontos als Datei-Container“ eine später seitens der Beklagten erfolgte Übermittlung einer einzigen PDF-Datei als den Urteilstenor des Landgerichts vollständig erfüllend ansah. Nach ihrem bisherigen Standpunkt und Vorbringen konnte ein solcher Datei-Container vielmehr allenfalls ausreichend sein, wenn er dieselbe Funktionalität aufwies wie das Benutzerkonto selbst, d.h. dieses nicht nur in seinen Inhalten, sondern auch seinen Funktionalitäten vollständig abbildete (vgl. dazu nachfolgend unter b aa). Soweit die Klägerin im Anschluss auf Seite 11 der Berufungserwiderung zu erkennen gegeben hat, dass sie nicht die Fortführung des Benutzerkontos begehrt, kann hieraus ebenfalls nicht geschlossen werden, dass sie nur die Kenntnisnahme von dessen Kommunikationsinhalten verlangt. Vielmehr hat sie dort zugleich ausgeführt, dass es ihr um den Zugriff auf das Nutzerprofil gehe. Auf Seite 16 desselben Schriftsatzes hat sie sodann geltend gemacht, dass es keine Rechtsgrundlage für die Sperrung des F.-Profils gebe. Auch hieraus wird deutlich, dass sie wie bereits erstinstanzlich weiterhin die Entsperrung des Nutzerprofils für die Erben begehrt hat.

Soweit die Klägerin schließlich in ihrer Revisionsbegründung (S. 42) vorgetragen hat, sie mache für die Erbengemeinschaft einen Anspruch auf Zugang zu dem Benutzerkonto bei der Beklagten geltend, um nähere Kenntnisse vom Inhalt des Kontos zu erlangen, hat der Senat dies im Revisionsverfahren nicht im Sinne einer Beschränkung des Klagebegehrens auf die Kenntniserlangung vom Kontoinhalt verstanden. Vielmehr differenziert die Klägerin mit dieser Formulierung zwischen dem von ihr geltend gemachten Anspruch einerseits (Zugang zu dem Benutzerkonto) und dessen Zweck andererseits (Kenntniserlangung vom Inhalt des Benutzerkontos). Das Motiv einer Klage darf aber nicht mit dem Inhalt des Klagebegehrens verwechselt werden.

(2) Auch aus den Entscheidungsgründen des Senatsurteils vom 12. Juli 2018 ergibt sich eindeutig, dass der Zugang zu dem vollständigen Benutzerkonto, zu dessen Gewährung die Schuldnerin verurteilt worden ist, die Möglichkeit der Gläubigerin mitumfasst, vom Benutzerkonto und dessen Inhalt auf dieselbe Art und Weise Kenntnis nehmen zu können, wie dies die Erblasserin konnte, und dass sich die Gläubigerin in dem Benutzerkonto mit Ausnahme einer aktiven Nutzung so „bewegen“ können muss wie zuvor die Erblasserin selbst.

(a) Dies ist bereits an der zahlreich im Senatsurteil erfolgenden Bestimmung der Verpflichtung der Schuldnerin zur kumulativen Gewährung sowohl des Zugangs zum vollständigen Benutzerkonto als auch zu dessen Kommunikationsinhalten erkennbar (vgl. Senatsurteil vom 12. Juli 2018 aaO Rn. 17, 18, 21, 28, 29, 31, 38, 54 und 78; ähnlich bereits oben unter bb zur Auslegung des Tenors des Vollstreckungstitels).

Vor allem aber ergibt sich eine solche Auslegung des Senatsurteils aus seiner erbrechtlichen Herleitung des Anspruchs der Gläubigerin (vgl. hierzu Pruns aaO S. 249 und Biermann aaO; zur erbrechtlichen Begründung bereits des Urteils des Landgerichts siehe oben unter (1) (a)). Der Senat hat ausgeführt, das Vertragsverhältnis sei mit seinen Rechten und Pflichten mit dem Tod der Erblasserin auf die Erben übergegangen, die hierdurch in dieses eingetreten seien und deshalb als Vertragspartner und neue Kontoberechtigte einen Primärleistungsanspruch auf Zugang zu dem Benutzerkonto der Erblasserin sowie den darin enthaltenen digitalen Inhalten hätten (Senatsurteil vom 12. Juli 2018 aaO Rn. 21 ff, $44,50,78,91)$.

- Aus dieser Stellung der Erben und dem auf sie übergegangenen Hauptleistungsanspruch der Erblasserin aus dem mit der Beklagten bestehenden Vertragsverhältnis folgt ohne weite- 
res, dass den Erben auf dieselbe Art und Weise Zugang zu dem Benutzerkonto zu gewähren ist wie zuvor der Erblasserin.

Einen Unterschied zwischen den diesbezüglichen Rechten der Erben und der Erblasserin gibt es mit Ausnahme der aktiven Nutzung nicht.

Dementsprechend hat der Senat an anderer Stelle ausgeführt, das Versetzen in den Gedenkzustand führe dazu, dass die wesentlichen Rechte aus dem Vertragsverhältnis, nämlich der Zugang zu dem Benutzerkonto, der Zugriff auf die dort gespeicherten Inhalte und die Verfügungsbefugnis hierüber, entfielen, so dass die Erreichung des Vertragszwecks nicht mehr möglich sei (Senatsurteil vom 12. Juli 2018 aaO Rn. 31). Auch hieraus wird deutlich, dass der Senat unter dem „Zugang zu dem Benutzerkonto“ einen solchen verstanden hat, wie er der Erblasserin möglich war, nämlich im Wege des aktiven "Zugriffs" auf die dort (und nicht auf einem anderen Medium) gespeicherten Inhalte, und wie er infolge des Versetzens des Kontos in den Gedenkzustand jetzt nicht mehr möglich ist.

Dieses Verständnis des Senats zeigt sich auch in seinen Ausführungen zum Schutz des Fernmeldegeheimnisses. Danach erfolgt die Zugangsgewährung für den Erben im Rahmen des unterstellt durch das Fernmeldegeheimnis geschützten Kommunikationsvorgangs. Der Erbe werde mit dem Tod des ursprünglichen Kontoberechtigten als neuer Vertragspartner und Kontoberechtigter zum Teilnehmer der auf Grund der Speicherung und Bereitstellung der Inhalte für das Benutzerkonto fortlaufenden Kommunikationsvorgänge (Urteil vom 12. Juli 2018 aaO Rn. 60). Eine Zugangsgewährung im Rahmen eines Kommunikationsvorgangs ist indes eine solche, wie sie auch gegenüber der Erblasserin im Wege des Einloggens in das Benutzerkonto mit den Zugangsdaten erfolgte.

Zum Datenschutz hat der Senat ausgeführt, mit dem Zugang zum Benutzerkonto erhalte der Erbe die Möglichkeit, auf die Kommunikation oder die mit dem Erblasser geteilten Bilder oder sonstigen Inhalte zuzugreifen. Die Beklagte sei in den Kommunikationsprozess insoweit eingebunden, als sie die Nachrichten zum Abruf für das Empfängerkonto bereitstelle sowie den Zugriff auf die geteilten Inhalte ermögliche und die entsprechende Plattform zur Verfügung stelle (Urteil vom 12. Juli 2018 aaO Rn. 69). Der hier beschriebene Zugang zum Benutzerkonto zur Ermöglichung des „Zugriffs" auf dessen Inhalte unter Inanspruchnahme der Plattform der Beklagten entspricht der Art und Weise des Zugangs, wie ihn die Erblasserin hatte. Dementsprechend ist die Beklagte nach der Entscheidung des Senats verpflichtet, den Abruf der auf ihrem Server gespeicherten Nachrichten dauerhaft zu ermöglichen, wobei der Tod des ursprünglich Berechtigten hieran nichts ändert, da das Konto nach dem Erbfall fortbesteht und der Erbe damit Berechtigter wird (Urteil vom 12. Juli 2018 aaO Rn. 73).

(b) Die Auffassung des Beschwerdegerichts, aus der Begründung des Senatsurteils vom 12. Juli 2018 ergebe sich, dass die Verpflichtung der Schuldnerin sich darin erschöpfe, der Gläubigerin Kenntnis vom Inhalt der im Benutzerkonto vorgehaltenen Inhalte zu vermitteln, trifft nicht zu.

Dies gilt zunächst für die vom Beschwerdegericht herangezogene Textstelle in Randnummer 36 des Senatsurteils, wonach Gegenstand des Rechtsstreits lediglich die Bereitstellung der vorhandenen Kontoinhalte zum Abruf durch die Erben ist.
- Mit dieser Formulierung hat der Senat allein die aktive Weiternutzung des Benutzerkontos durch die Erben vom Streitgegenstand des Rechtsstreits abgegrenzt, der eine solche aktive Weiternutzung nicht umfasst.

Erörtert wird dort eine eventuelle Unvererbbarkeit des Vertragsverhältnisses. Diese könnte wegen deren höchstpersönlicher Natur allenfalls im Hinblick auf eine aktive Nutzung des Kontos gegeben sein. Aus den Ausführungen des Senats kann dagegen nicht geschlossen werden, dass die Nutzung des Kontos im Übrigen nicht dergestalt zu ermöglichen ist, wie sie auch der Erblasserin zur Verfügung zu stellen war (so auch Pruns aaO S. 248).

[...]

(c) Aus den von der Rechtsbeschwerdeerwiderung darüber hinaus herangezogenen Textstellen des Senatsurteils vom 12. Juli 2018 ergibt sich ebenfalls nicht, dass sich die Verpflichtung der Schuldnerin auf die bloße Vermittlung der Kommunikationsinhalte des Benutzerkontos beschränkt.

[...]

b) Die Schuldnerin hat ihre Verpflichtung aus dem rechtskräftigen Urteil des Landgerichts vom 17. Dezember 2015, der Gläubigerin Zugang zu dem vollständigen Benutzerkonto und den darin vorgehaltenen Kommunikationsinhalten zu gewähren, nicht erfüllt. Sie hat der Gläubigerin nicht die Möglichkeit eingeräumt, vom Benutzerkonto und dessen Inhalt auf dieselbe Art und Weise Kenntnis nehmen zu können, d.h. sich in dem Benutzerkonto mit Ausnahme einer aktiven Nutzung so „bewegen“ zu können wie zuvor die Erblasserin selbst. Es kann auch nicht von einer Einigung der Parteien dergestalt ausgegangen werden, dass mit der Übersendung des USB-Sticks an die Gläubigerin durch die Schuldnerin am 30. August 2018 deren Verpflichtung aus dem Vollstreckungstitel erfüllt werden konnte.

aa) Die Rechtsbeschwerdeerwiderung (S. 20) räumt ein, mit der Überlassung des USB-Sticks der Gläubigerin keinen „direkten“ Zugang zum Benutzerkonto gewährt zu haben. Letzteres ist indes wie ausgeführt zur Erfüllung der titulierten Forderung der Gläubigerin erforderlich. Ob dieser Verpflichtung überhaupt mittels der Übergabe eines USB-Sticks nachgekommen werden kann, bedarf vorliegend keiner Entscheidung.

- Denkbar wäre das allenfalls, wenn durch die auf dem Stick befindlichen Dateien das Benutzerkonto vollständig und originalgetreu abgebildet werden würde einschließlich nicht nur der Darstellung seiner Inhalte, sondern auch der Eröffnung aller seiner Funktionalitäten mit Ausnahme derer, die seine aktive Weiternutzung betreffen und der (deutschen) Sprache, in der das Benutzerkonto zu Lebzeiten der Erblasserin vertragsgemäß geführt wurde.

Die Schuldnerin hat einen solchen Inhalt des von ihr übergebenen USB-Sticks, auf dem sich unstreitig nur eine einzige PDFDatei befindet, nicht vorgetragen. Im Übrigen ergibt sich ein fehlendes originalgetreues Abbild von dem Benutzerkonto bereits daraus, dass die Inhalte der vorgenannten PDF-Datei im Unterschied zu dem Benutzerkonto selbst zum Teil in englischer Sprache gehalten sind.

bb) Es kann auch nicht von einer Einigung der Parteien dahingehend ausgegangen werden, dass die Verpflichtung aus dem Vollstreckungstitel dadurch erfüllt werden kann, dass die Schuldnerin dem Gläubigervertreter einen USB-Stick übersendet, auf dem die Inhalte des Benutzerkontos gespeichert sind. [...]

c) Der angefochtene Beschluss erweist sich auch nicht aus anderen Gründen als richtig ( $\$ 577$ Abs. 3 ZPO). [...] 
aa) Die Rechtsbeschwerdeerwiderung macht unter Berufung auf entsprechenden Instanzvortrag der Schuldnerin geltend, gegenwärtig gebe es keinen „read only“-Zugang. Das Netzwerk „F.“ sei so aufgebaut, dass mit dem „Einloggen“ und der Kontonutzung automatische Prozesse aktiviert würden, etwa der Versand einer Mitteilung an Freunde, dass der jeweilige Nutzer wieder aktiv sei. Dies umfasse automatische Erinnerungen an den Geburtstag der verstorbenen L. W. an ihre „Freunde“ und automatische Vorschläge an andere Nutzer, die Verstorbene zum „Freund“ zu machen. Diejenigen, die die Verstorbene gekannt hätten, wären dadurch innerlich tief und negativ berührt. Die Schuldnerin zu verpflichten, den F.-Dienst so zu verändern, dass Nutzer unerwartete und verstörende Nachrichten wie Freundschaftsanfragen von verstorbenen Nutzern erhielten, sei jedenfalls dann unzumutbar, wenn - wie vorliegend - eine Überlassung gut strukturierter und durchsuchbarer Daten per Memory-Stick ohne direkten Zugang zum Konto der L. W. dem Interesse der Gläubigerin gerecht werde, von den Inhalten des Kontos Kenntnis zu nehmen. Eine Verpflichtung der Schuldnerin, einen nur lesenden Zugang („read only“-Zugang) zu dem Konto selbst zu schaffen, stehe außer Verhältnis, zumal nicht einmal sicher sei, ob ein solcher Zugang technisch eingerichtet werden könne.

Ebenso wenig könne einfach der Gedenkzustand aufgehoben werden. Dies führe dazu, dass der Gläubigerin die aktive Nutzung des Kontos der Erblasserin unter deren Namen möglich wäre, was den vertraglichen Bestimmungen widerspreche. Zudem würden die Kommunikationspartner der Erblasserin in diesem Fall Nachrichten erhalten, aus denen sich ergäbe, dass diese wieder aktiv sei. Die Aktivierung der Benutzerkonten von Verstorbenen könne zu erheblichen Irritationen, Bestürzung und traumatischen Erfahrungen bei anderen Mitgliedern der F.-Gemeinschaft führen. Aus den vorgenannten Gründen liege in Forderungen der Gläubigerin, die über die Vermittlung der in dem Konto der Erblasserin vorhandenen Informationen hinausgingen, eine unzulässige Rechtsausübung.

bb) Dem ist nicht zu folgen. Es kann weder von einer Unmöglichkeit noch von einer Unzumutbarkeit oder Unverhältnismäßigkeit der Erfüllung der titulierten Verpflichtung der Schuldnerin ausgegangen werden.

(1) Dabei kann unterstellt werden, dass es derzeit zu den Benutzerkonten des Netzwerks der Beklagten keinen „read only“-Zugang gibt, d.h. einen auf die übliche Weise mittels der Zugangsdaten erfolgenden Zugang zu dem Benutzerkonto, der lediglich die aktive Nutzung des Kontos nicht zulässt. Maßgeblich ist vorliegend allein, ob die Schuldnerin in der Lage ist, einen Zugang - gegebenenfalls erstmals - herzustellen, mittels dessen Gewährung sie ihre Verpflichtung aus dem Vollstreckungstitel erfüllen kann. Die Rechtsbeschwerdeerwiderung trägt in diesem Zusammenhang nicht mit der erforderlichen Bestimmtheit vor, dass es technisch nicht möglich ist, einen „read only“-Zugang in vorgenanntem Sinne einzurichten (zum Unmöglichkeitseinwand im Zwangsvollstreckungsverfahren vgl. BGH, Beschluss vom 27. November 2008 - I ZB 46/08, NJW-RR 2009, 443 Rn. 13; MüKoZPO/Gruber, 5. Aufl., $\$ 888$ Rn. 13). Auch aus dem von ihr in Bezug genommenen Sachvortrag der Schuldnerin ergibt sich dies nicht.

- (2) Letztlich kommt es hierauf jedoch nicht an. Denn die Einrichtung eines „read only“-Zugangs ist nur eine von mehreren Handlungen, mittels derer die Schuldnerin ihre Verpflichtung aus dem Vollstreckungstitel erfüllen kann.

Dies kann etwa auch durch die Aufhebung des Gedenkzustandes erfolgen, durch den der Gläubigerin derzeit der Zugang zu dem Benutzerkonto verwehrt wird. Soweit ihr hierdurch ein über den titulierten Anspruch hinausgehender Zugang gewährt werden würde, der es ihr faktisch ermöglichen würde, das Benutzerkonto auch aktiv weiterzunutzen, ist dies - worauf die Rechtsbeschwerdebegründung zutreffend hinweist - vollstreckungsrechtlich unbedenklich. Die Gläubigerin wäre zu einer solchen weitergehenden Nutzung des Kontos nicht aufgrund des Vollstreckungstitels berechtigt. Ist die Schuldnerin der Auffassung, dass die Gläubigerin zu einer derartigen Nutzung auch materiell-rechtlich nicht berechtigt ist, kann sie von ihr Unterlassung verlangen.

- Im Übrigen sind keine Anhaltspunkte dafür ersichtlich, dass die Gläubigerin im Falle der vollumfänglichen Zugangsgewährung das Benutzerkonto aktiv weiternutzen würde. Weder hat sie dies angekündigt noch ist ein Interesse ihrerseits hieran ersichtlich.

(3) Der Schuldnerin ist die Erfüllung ihrer Verpflichtung aus dem Vollstreckungstitel im Wege der Aufhebung des Gedenkzustandes auch nicht - unabhängig von der Frage der Zulässigkeit eines solchen Einwands im Vollstreckungsverfahren (verneinend MüKoZPO/Gruber aaO Rn. 14 mwN) - aus den von ihr angeführten Gründen unzumutbar. Denn sie könnte - worauf die Rechtsbeschwerdebegründung ebenfalls zutreffend hinweist - das Benutzerkonto umbenennen und hierdurch oder auf andere Weise gegenüber den Nutzern des Netzwerks kenntlich machen, dass das Konto nicht mehr von der Erblasserin, sondern von deren Erben genutzt wird und daher etwaige automatisch generierte Meldungen nicht von der Erblasserin stammen oder durch deren Kontonutzung angestoßen worden sind. Bei einer solchen Verfahrensweise droht der Schuldnerin auch kein unverhältnismäßiger Nachteil (zur Geltung des Grundsatzes der Verhältnismäßigkeit im Zwangsvollstreckungsverfahren vgl. BGH, Beschluss vom 20. Dezember 2006 - VII ZB 88/06, NJW 2007, 1276 Rn. 21). [...] 